\title{
CATALISADORES DE ÓXIDO DE FERRO DOPADOS COM METAIS DE TRANSIÇÃO APLICADOS NA OZONIZAÇÃO CATALÍTICA DO CORANTE SAFIRA PROCION
}

\author{
J. SUAVE ${ }^{1}$ L. B. LEME$^{1}$ e R. F. P. M. MOREIRA ${ }^{1}$ \\ ${ }^{1}$ Universidade Federal de Santa Catarina, Departamento de Engenharia Química e Engenharia de \\ Alimentos \\ E-mail para contato: j.suave@ posgrad.ufsc.br
}

\begin{abstract}
RESUMO - Catalisadores baseados em óxidos de ferro têm recebido atenção por apresentarem elevada atividade catalítica em reações de oxidação avançada. O lodo químico produzido no tratamento ativo da drenagem ácida de mina foi utilizado como precursor no preparo de catalisadores de hematita pura ou dopada com diferentes proporções de cobre, manganês, prata ou zinco. Os catalisadores foram caracterizados e a atividade catalítica foi determinada pela mineralização do corante têxtil safira procion H-EXL em reator batelada com aplicação constante de ozônio. Os resultados indicaram que a ozonização catalítica com a hematita é mais eficiente para a mineralização do corante do que somente a ozonização. A dopagem da hematita produz catalisadores com atividade catalítica superior $(\mathrm{Mn}$ e $\mathrm{Cu})$ ou inferior (Ag e $\mathrm{Zn}$ ) à hematita pura. $\mathrm{O}$ teor de metal impregnado também modifica a atividade catalítica, sendo que o catalisador dopado com $10 \%$ de manganês em massa apresenta os melhores resultados.
\end{abstract}

\section{INTRODUÇÃO}

A drenagem ácida de mina (DAM) é um importante problema de poluição ambiental gerado pela indústria de mineração. Quando os rejeitos do beneficiamento de carvão ricos em sulfetos de ferro são expostos à atmosfera, em presença de água, ocorre oxidação que produz ácido sulfúrico e libera metais pesados e outros poluentes para a drenagem (Sheoran e Sheoran, 2006). Entretanto, a DAM pode ser neutralizada usando cal, cal hidratada, soda caústica, entre outros, o que resulta na produção de um lodo químico volumoso, cuja disposição final representa mais um problema ambiental e custo adicional. Apesar disso, é possível reaproveitar o lodo químico, rico em metais como ferro e manganês, para produzir novos materiais com valor agregado, como adsorventes (Flores et al., 2012), pigmentos (Kirby et al., 1999; Marcello et al., 2008), coagulantes (Ruihua et al., 2011) e catalisadores (Andersen et al., 2012; Flores et al., 2012).

Catalisadores baseados em óxidos de ferro têm recebido atenção nos últimos anos por apresentarem elevada atividade catalítica em processos de oxidação avançada. Além disso, possuem excelente estabilidade e custo-efetividade, elevada resistência à corrosão e propriedades ambientalmente amigáveis que preenchem muitos requisitos de um bom catalisador (Pradhan e Parida, 2012). Alguns estudos têm mostrado resultados satisfatórios na utilização de óxidos de ferro para tratar efluentes líquidos através do processo de ozonização catalítica. Zhang e Ma (2008) reportaram que a goetita $(\mathrm{FeOOH})$ aumenta substancialmente a degradação do 
nitrobenzeno comparado apenas à ozonização sozinha. Jung et al. (2008) promoveram a degradação do ácido p-clorobenzóico utilizando magnetita comercial e biogênica $\left(\mathrm{Fe}_{3} \mathrm{O}_{4}\right)$. Lv et al. (2010) usaram a maghemita $\left(\gamma-\mathrm{Fe}_{2} \mathrm{O}_{3}\right)$ codopada com cobalto e manganês para alcançar a mineralização efetiva do ácido 2,4-diclorofenoxiacético. A introdução de manganês aumentou a quantidade de grupos hidroxila superficiais e a quantidade de água quimissorvida, bem como, aumentou a transferência interfacial de elétrons, resultando em uma atividade catalítica maior.

A ozonização catalítica foi desenvolvida para ultrapassar as limitações dos processos de ozonização, tais como a formação de subprodutos e as reações seletivas do ozônio. Segundo Moussavi et al. (2012), um catalisador pode melhorar as reações de ozonização através de diferentes modos: (i) o ozônio pode reagir com os grupos funcionais presentes na superfície do catalisador e gerar espécies oxidantes mais reativas que o ozônio molecular; (ii) o catalisador pode fornecer uma superfície para a reação do ozônio molecular com o composto alvo; (iii) o catalisador pode adsorver o composto que finalmente, reage com o ozônio dissolvido.

No presente trabalho, catalisadores de hematita $\left(\alpha-\mathrm{Fe}_{2} \mathrm{O}_{3}\right)$ obtidos a partir do lodo químico produzido no tratamento ativo da DAM foram preparados e avaliados na ozonização catalítica do corante têxtil safira procion H-EXL. Na tentativa de aperfeiçoar o desempenho da hematita, esta foi dopada com os metais de transição cobre, manganês, prata ou zinco em diferentes proporções.

\section{MATERIAS E MÉTODOS}

\subsection{Materiais}

Os materiais utilizados neste trabalho foram goetita acicular proveniente do tratamento ativo da DAM fornecida pela Carbonífera Criciúma S.A, nitrato de cobre II trihidratado (Vetec), nitrato de manganês II hidratado (Vetec), nitrato de prata (Nuclear), nitrato de zinco hexahidratado (Vetec) e o corante reativo azo aniônico safira procion H-EXL (Basf).

\subsection{Preparação dos catalisadores}

A Tabela 1 relaciona todos os catalisadores preparados e suas respectivas denominações.

Tabela 1 - Catalisadores utilizados na ozonização catalítica e suas respectivas denominações

\begin{tabular}{ccc}
\hline Catalisador & $\begin{array}{c}\text { Porcentagem Teórica } \\
\text { de Metal Dopado }\end{array}$ & Denominação \\
\hline Goetita acicular & - & $\mathrm{GA}$ \\
Hematita & - & $\mathrm{H}$ \\
& $5 \%$ & $\mathrm{HCu}-5$ \\
Hematita dopada com cobre & $2,5 \%$ & $\mathrm{HMn}-2,5$ \\
& $5 \%$ & $\mathrm{HMn}-5$ \\
Hematita dopada com manganês & $10 \%$ & $\mathrm{HMn}-10$ \\
& $20 \%$ & $\mathrm{HMn}-20$ \\
Hematita dopada com prata & $5 \%$ & $\mathrm{HAg}-5$ \\
Hematita dopada com zinco & $5 \%$ & $\mathrm{HZn}-5$ \\
Hematita codopada com cobre e manganês & $2,5 \%(\mathrm{Cu})+2,5 \%(\mathrm{Mn})$ & $\mathrm{HCu} / \mathrm{Mn}-2,5$ \\
\hline
\end{tabular}


Os catalisadores foram preparados a partir da goetita acicular, cuja síntese está bem descrita em Andersen et al. (2012). Para a dopagem dos catalisadores com os diferentes metais foi utilizado o método de impregnação por via úmida. Cerca de $5 \mathrm{~g}$ de goetita foram imersos em $150 \mathrm{~mL}$ de solução aquosa contendo o nitrato do metal de transição a ser impregnado com concentração adequada para se obter a quantidade mássica desejada do metal. As dispersões foram mantidas sob agitação vigorosa por 24 horas e, posteriormente, secas a $110^{\circ} \mathrm{C}$ por 48 horas, moídas e peneiradas $(<0,149 \mathrm{~mm})$. Finalmente, o sólido obtido foi calcinado a $450^{\circ} \mathrm{C}$ por 4 horas em atmosfera de ar, com taxa de aquecimento de $10^{\circ} \mathrm{C} / \mathrm{min}$. Um catalisador sem impregnação de metal (hematita pura) também foi preparado, aplicando o mesmo tratamento térmico.

\subsection{Caracterização dos catalisadores}

O precursor goetita, a hematita e os catalisadores dopados foram caracterizados por difração de raios-X (DRX), análise termogravimétrica (TGA) e determinação de área superficial BET. Os difratogramas foram obtidos em um difratômetro X'Pert (Philips) empregando radiação de $\mathrm{Cu} \mathrm{Ka}$ e intervalo de varredura de 10 a $80^{\circ}(2 \theta)$. As análises de TGA foram realizadas em um equipamento DTG60/60H (Shimadzu). As amostras foram aquecidas da temperatura ambiente até $950^{\circ} \mathrm{C}$ com taxa de aquecimento de $10^{\circ} \mathrm{C} / \mathrm{min}$ em atmosfera de nitrogênio. A determinação da área superficial BET foi feita em um equipamento Autosorb-1 (Quantachrome Instruments).

\subsection{Ensaios de ozonização catalítica}

Os ensaios de ozonização catalítica foram conduzidos em um reator cilíndrico de vidro com volume útil de 1,5 L. Em cada ensaio, $500 \mathrm{mg} / \mathrm{L}$ de catalisador e $1 \mathrm{~L}$ de solução de safira procion H-EXL na concentração de $200 \mathrm{mg} / \mathrm{L}$ eram adicionados ao reator. A reação iniciava-se pela aplicação de ozônio, o qual era gerado a partir do ar ambiente em um ozonizador ID-10 (O3R Philozon) provido com concentrador de oxigênio. As reações eram realizadas em temperatura ambiente $\left(25^{\circ} \mathrm{C}\right)$ e mantidas constantemente sob agitação e borbulhamento de ozônio. Amostras eram retiradas em intervalos pré-determinados e filtradas em membrana de PVDF (Millipore) com porosidade de $0,22 \mu \mathrm{m}$ e, em seguida, analisadas. Medidas de carbono orgânico total (COT) e nitrogênio total (NT) foram obtidas simultaneamente em um analisador TOC-VCPH (Shimadzu) equipado com um amostrador automático ASI-V e combinado a um módulo de nitrogênio TNM-1. Já as medidas de condutividade foram feitas em um condutivímetro DM-32 (Digimed).

\section{RESULTADOS E DISCUSSÃO}

\subsection{Caracterização dos catalisadores}

Os resultados das análises de DRX do precursor goetita e do catalisador hematita são apresentados na Figura 1 (a). Os difratogramas indicam que o tratamento térmico a $450^{\circ} \mathrm{C}$ transformou toda a fase cristalina goetita para hematita, já que após o tratamento térmico apenas os picos característicos da hematita são observados (Kirby et al., 1999; Deraz e Alarif, 2012). Com relação aos catalisadores dopados foi notado que a presença dos metais de transição nas quantidades adicionadas não interferiu significativamente na estrutura cristalina ou no grau de cristalinidade da hematita. Entretanto, conforme Figura 1 (b), nos catalisadores preparados com mais de $10 \%$ de manganês é possível observar o aparecimento de picos referentes ao $\mathrm{MnO}_{2}$ (Andersen et al., 2012; Rezaei et al., 2013). 


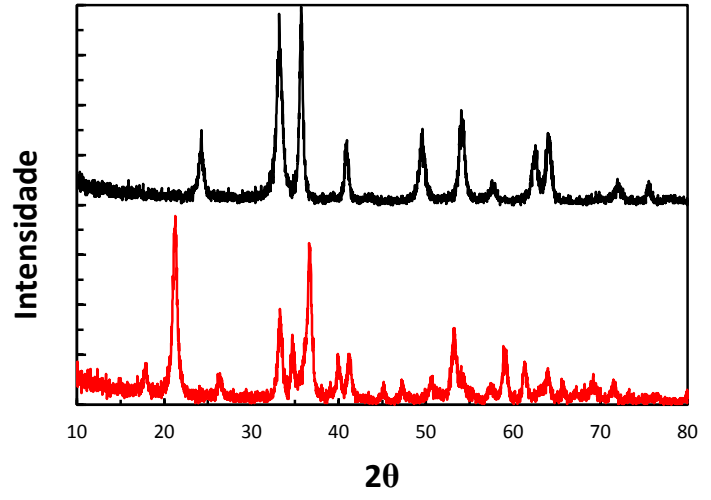

(a)

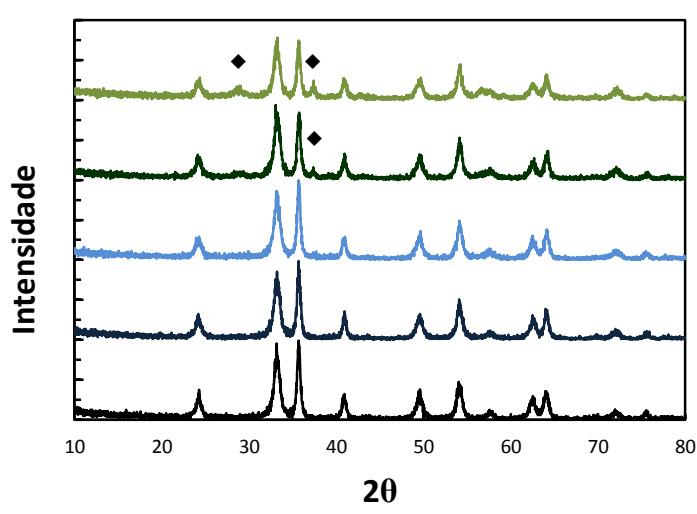

(b)

Figura 1 - (a) Difratogramas de raios-X da goetita acicular (-) e da hematita (-); (b) Difratogramas de raios- $X$ dos catalisadores dopados com diferentes concentrações de manganês em massa: hematita (-), HMn-2,5 (-) ,HMn-5 (-), HMn-10 (-) e HMn-20 (-), sendo $\bullet=\mathrm{MnO}_{2}$.

Na Figura 2 são apresentados os resultados da análise térmica para amostras de goetita acicular e hematita. A hematita não apresentou perda de massa significativa durante toda a faixa de temperatura estudada, enquanto que a goetita exibiu uma perda de massa de cerca de $8 \%$ entre 200 e $300^{\circ} \mathrm{C}$, que se deve à dehidroxilação térmica e a formação de uma nova fase cristalina (Zhang e Ma, 2008). O pico endotérmico observado em $277^{\circ} \mathrm{C}$ representa a transformação de fase.

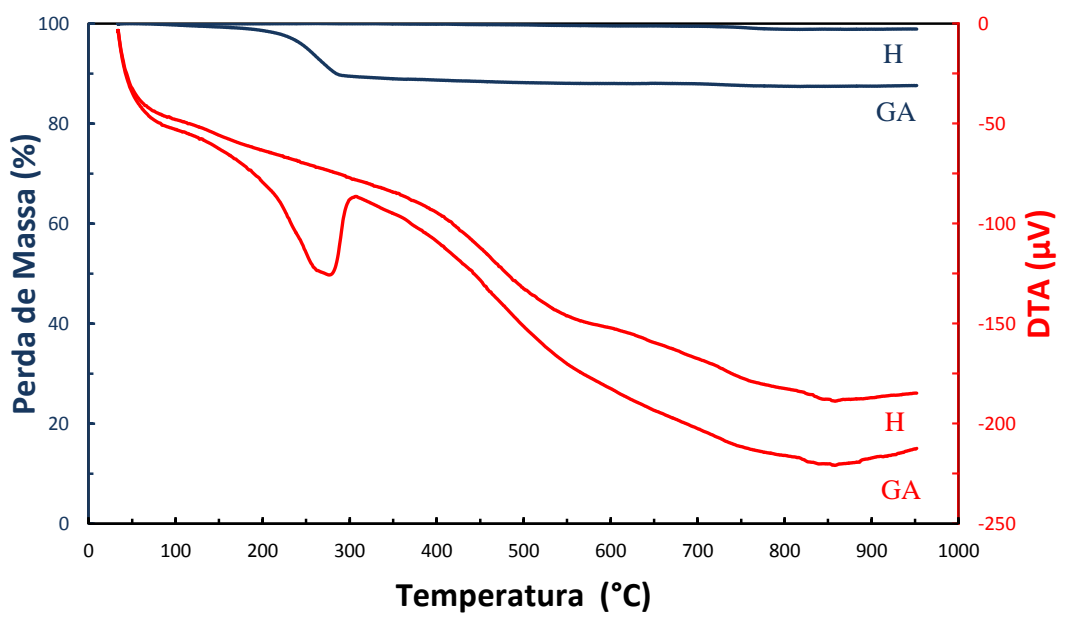

Figura 2 - Termogramas da goetita acicular (GA) e da hematita $(\mathrm{H})$.

As análises térmicas dos diversos catalisadores dopados indicaram que não há diferenças pronunciadas no comportamento térmico das amostras dopadas em comparação com a hematita pura. Em relação à série de catalisadores dopados com concentrações variáveis de manganês, notou-se que a partir da concentração de $10 \%$ de $\mathrm{Mn}$, a perda de massa começou a ser levemente favorecida. Ocorreu uma perda de massa de 1\% para HMn-10\% e de 2\% para HMn-20\%. Logo, o aumento do teor de manganês impregnado tende a afetar o comportamento térmico da hematita. 
A Tabela 2 fornece as áreas superficiais BET determinadas para cada um dos catalisadores sintetizados. Valores de volume total de poros e diâmetro médio de poros também são apresentados para alguns dos catalisadores. Observa-se que a área superficial da goetita reduziu cerca de $35 \%$ com a transformação térmica para a fase hematita. A dopagem dos catalisadores também contribuiu para uma redução ainda maior da área BET, exceto no caso do catalisador dopado com 5\% de Mn que apresentou uma área superficial maior do que a própria hematita pura. Contudo, o aumento na adição de Mn também contribui para a diminuição da área superficial. Em termos gerais, os resultados sugerem que a transformação térmica da goetita e a dopagem (10\% de $\mathrm{Mn}$ ) favorecem a redução do volume total de poros e o aumento do diâmetro médio dos poros.

Tabela 2 - Área superficial BET, volume total de poros e diâmetro médio de poros para os diversos catalisadores preparados

\begin{tabular}{cccc}
\hline Catalisador & Área BET $\left(\mathbf{m}^{\mathbf{2}} \mathbf{g}\right)$ & $\begin{array}{c}\text { Volume Total de } \\
\text { Poros }\left(\mathbf{c m}^{\mathbf{3}} / \mathbf{g}\right)\end{array}$ & $\begin{array}{c}\text { Diâmetro Médio de } \\
\text { Poros }(\AA)\end{array}$ \\
\hline GA & 89,16 & 0,641 & 287,7 \\
H & 57,88 & 0,461 & 318,5 \\
HAg-5 & 41,53 & - & - \\
HCu-5 & 45,02 & - & - \\
HMn-2,5 & 58,72 & - & - \\
HMn-5 & 63,52 & - & - \\
HMn-10 & 51,05 & 0,439 & 344,0 \\
HMn-20 & 45,66 & - & - \\
HZn-5 & 38,38 & - & - \\
HCu/Mn-2,5 & 47,21 & - & - \\
\hline
\end{tabular}

\subsection{Avaliação da atividade catalítica}

Primeiramente, testes preliminares de adsorção indicaram que o corante safira procion $\mathrm{H}$ EXL não tende a ser adsorvido sobre as partículas de hematita na ausência de aplicação de ozônio. Em seguida, a atividade catalítica dos diversos catalisadores foi avaliada pela redução de COT durante um tempo total de 3 horas. A Figura 3 (a) apresenta as curvas obtidas pela ozonização catalítica com hematita e pela ozonização simples. Embora em 3 horas de reação, as porcentagens de remoção de COT sejam próximas, $51 \%$ para a ozonização e $55 \%$ para a ozonização catalítica (ver Tabela 3), a velocidade inicial de reação $\left(-r_{0}\right)$ para a ozonização catalítica $(0,67 \mathrm{mg} / \mathrm{L} \cdot \mathrm{min})$ é quase o dobro da velocidade inicial para a ozonização (0,36 mg/L.min).

Na tentativa de verificar se a degradação apresentada pela ozonização catalítica é devida à geração de espécies oxidantes mais reativas, como os radicais hidroxila ( $\mathrm{OH}$ ), foram executados experimentos utilizando terc-butanol. O terc-butanol é conhecido por ser um forte sequestrador de radicais ${ }^{\circ} \mathrm{OH}$, apresentando uma constante de velocidade de reação de $6 \times 10^{8} \mathrm{M}^{-1} \mathrm{~s}^{-1}$ com os radicais hidroxila e de apenas $3 \times 10^{-3} \mathrm{M}^{-1} \mathrm{~s}^{-1}$ com o ozônio (Lv et al., 2010). Segundo os resultados obtidos, inseridos também na Figura 3 (a), ambas a ozonização simples e a ozonização catalítica sofreram uma diminuição na degradação do corante quando o terc-butanol foi adicionado, indicando que os radicais ${ }^{\circ} \mathrm{OH}$ são as principais espécies ativas na degradação do safira procion. 


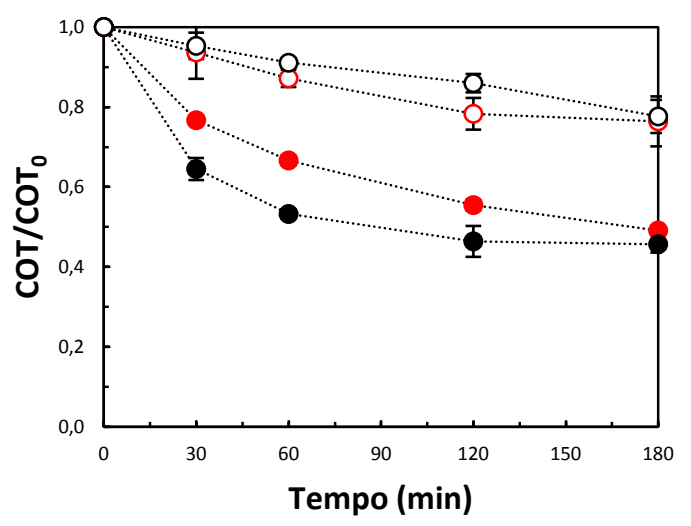

(a)

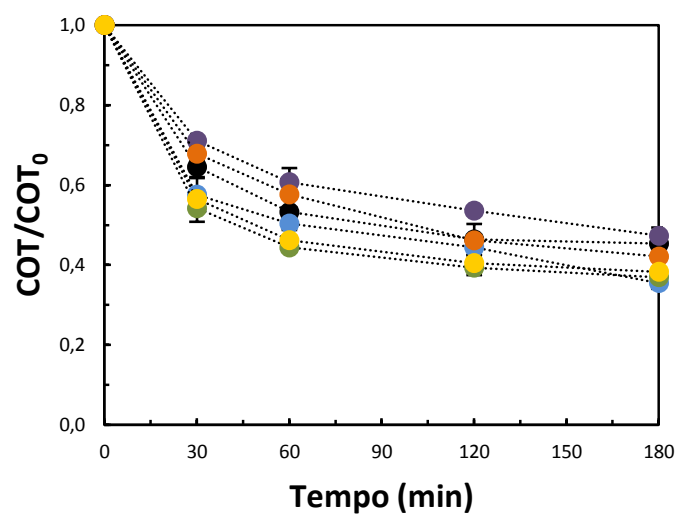

(b)

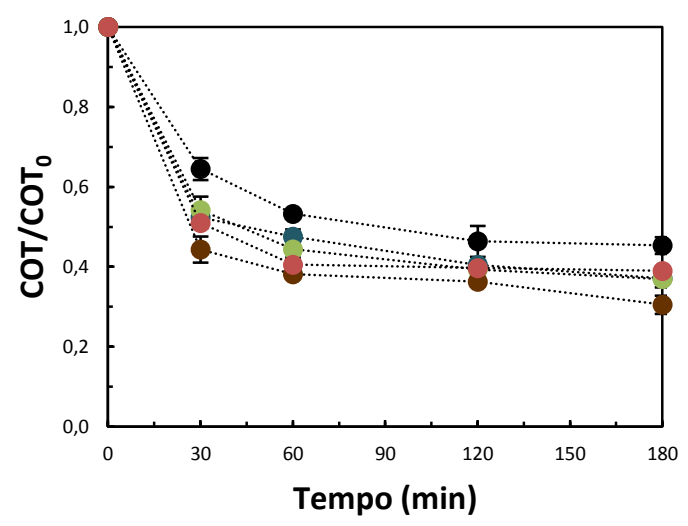

(c)

Figura 3 - (a) Remoção de COT pela ozonização simples e ozonização catalítica: $(\bullet) \mathrm{O}_{3},(\bullet)$ $\mathrm{H} / \mathrm{O}_{3}$, (०) $\mathrm{O}_{3} / t-\mathrm{BuOH}$ e (०) $\mathrm{H} / \mathrm{O}_{3} / t-\mathrm{BuOH}$; (b) Remoção de COT pela ozonização catalítica por

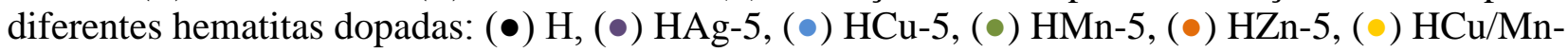
2,5. (c) Remoção de COT pela ozonização catalítica porm hematita dopada com diferentes concentrações de manganês: (•) H, (•) HMn-2,5, (•) HMn-5, (•) HMn-10 e (•) HMn-20. $[$ corante $]=200 \mathrm{mg} / \mathrm{L} ;$ catalisador $]=500 \mathrm{mg} / \mathrm{L}$; vazão de $\mathrm{O}_{3}=1 \mathrm{~L} / \mathrm{min} ;[t-\mathrm{BuOH}]=1,0 \mathrm{~g} / \mathrm{L}$.

Na Figura 3 (b) são apresentadas as remoções de COT utilizando a hematita dopada com 5\% de $\mathrm{Ag}, \mathrm{Cu}, \mathrm{Mn}$ ou $\mathrm{Zn}$ e codopada com 2,5/2,5\% de $\mathrm{Cu} / \mathrm{Mn}$. Observa-se que a prata e o zinco não melhoraram o desempenho da hematita, enquanto que o cobre e o manganês forneceram, respectivamente, um aumento de 16 e $15 \%$ na remoção de COT. Considerando os resultados de área superficial BET, nota-se que os catalisadores dopados com prata e zinco foram os que apresentaram a menor área superficial, assim, o desempenho inferior destes catalisadores pode estar associado também à diminuição da área BET. Entretanto, a velocidade inicial de reação quando foi utilizado o catalisador HMn-5 foi maior, conforme indica a Tabela 3.

O safira procion H-EXL é um corante azo aniônico caracterizado por apresentar o grupamento $(-\mathrm{N}=\mathrm{N}-)$ e também grupos contendo átomos de enxofre e sódio. Durante a mineralização da solução de corante pela ozonização catalítica torna-se possível a liberação dos átomos de nitrogênio, enxofre e sódio no meio aquoso na forma de compostos iônicos, aumentando, então, a condutividade da solução. Os resultados mostrados na Tabela 3 apontam um 
aumento acima de $100 \%$ na condutividade original da solução para a maioria dos experimentos realizados. Acrescenta-se ainda, que medidas de nitrogênio total sugerem que os átomos de nitrogênio inicialmente presentes nas moléculas de safira procion permanecem em solução após a degradação, possivelmente como íons $\mathrm{NO}_{3}{ }^{-}$e/ou $\mathrm{NH}_{4}{ }^{+}$.

Tabela 3 - Velocidade inicial de reação, remoção de COT (\%), acréscimo de condutividade (\%) e pH final, após 3 horas de ozonização com diferentes catalisadores

\begin{tabular}{ccccc}
\hline Catalisador & $\begin{array}{c}\text { Remoção de } \\
\text { COT }(\%)\end{array}$ & $\begin{array}{c}\text { Acréscimo de } \\
\text { Condutividade }(\%)\end{array}$ & pH Final & $\begin{array}{c}-\mathbf{r}_{\mathbf{0}} \\
(\mathbf{m g} / \mathbf{L} . \mathbf{m i n})\end{array}$ \\
\hline- & $51 \pm 0$ & $186 \pm 0$ & $2,7 \pm 0,1$ & $0,36 \pm 0,03$ \\
$\mathrm{H}$ & $55 \pm 2$ & $171 \pm 0$ & $2,4 \pm 0,1$ & $0,67 \pm 0,14$ \\
$\mathrm{HAg}-5$ & $53 \pm 2$ & $90 \pm 7$ & $2,6 \pm 0,0$ & $0,56 \pm 0,02$ \\
$\mathrm{HCu}-5$ & $64 \pm 1$ & $52 \pm 13$ & $2,3 \pm 0,0$ & $0,82 \pm 0,14$ \\
$\mathrm{HMn}-2,5$ & $63 \pm 1$ & $210 \pm 9$ & $2,6 \pm 0,0$ & $1,01 \pm 0,07$ \\
$\mathrm{HMn}-5$ & $63 \pm 2$ & $195 \pm 2$ & $2,6 \pm 0,2$ & $1,02 \pm 0,05$ \\
$\mathrm{HMn}-10$ & $69 \pm 2$ & $121 \pm 2$ & $2,4 \pm 0,0$ & $1,16 \pm 0,09$ \\
$\mathrm{HMn}-20$ & $61 \pm 1$ & $134 \pm 4$ & $2,6 \pm 0,0$ & $0,96 \pm 0,01$ \\
$\mathrm{HZn}-5$ & $58 \pm 3$ & $152 \pm 0$ & $2,7 \pm 0,0$ & $0,61 \pm 0,01$ \\
$\mathrm{HCu} / \mathrm{Mn}-2,5$ & $62 \pm 1$ & $129 \pm 10$ & $2,4 \pm 0,1$ & $0,82 \pm 0,05$ \\
\hline
\end{tabular}

[corante] $=200 \mathrm{mg} / \mathrm{L}$; [catalisador] $=500 \mathrm{mg} / \mathrm{L}$; vazão de $\mathrm{O}_{3}=1 \mathrm{~L} / \mathrm{min}$.

Uma solução de safira procion apresenta $\mathrm{pH}$ em torno de 3,0. A análise de $\mathrm{pH}$ das soluções ensaiadas indicou que o $\mathrm{pH}$ não se altera bruscamente com o tratamento, permanecendo entre 2,03,0 no final da reação (Tabela 3). Sabe-se que o ozônio em pH ácido se apresenta em sua forma molecular no meio aquoso, mas em $\mathrm{pH}$ alcalino reage com íons hidroxila para produzir radicais 'OH (Von Gunten, 2003). Considerando que o pH reacional é ácido durante todo o período de reação, deduz-se que o ozônio deve se decompor na superfície dos catalisadores para então, produzir os radicais hidroxila que forneceriam a melhora no tratamento por ozonização catalítica.

Na Figura 3 (c) e ainda na Tabela 3 são mostrados os resultados obtidos nos ensaios de degradação utilizando a série de catalisadores preparados com diferentes teores de manganês. Primeiramente, observa-se que os resultados de remoção de COT alcançados pelo emprego dos catalisadores HMn-2,5 e HMn-5 são semelhantes. Porém, o catalisador com $10 \%$ de manganês apresentou resultados ligeiramente superiores, enquanto que o catalisador com $20 \%$ de manganês teve desempenho menor do que todos os outros catalisadores com manganês estudados. Isto sugere que há uma concentração ótima de inserção de metal na rede cristalina da hematita para a obtenção de resultados melhorados.

\section{CONCLUSÕES}

O catalisador hematita preparado a partir de goetita proveniente do tratamento ativo da DAM é capaz de aumentar a mineralização do corante têxtil safira procion H-EXL obtida pelo tratamento de ozonização simples. A dopagem da hematita com metais de transição através do método de impregnação por via úmida produz catalisadores com atividade catalítica superior (Mn e $\mathrm{Cu}$ ) ou inferior ( $\mathrm{Ag}$ e $\mathrm{Zn}$ ) à hematita pura. A ordem de atividade catalítica encontrada foi HMn- 
$10>$ HMn-5 > HMn-2,5 > HMn-20 > HCu-5 = HCu/Mn-2,5 > H > HZn-5 > HAg-5 > ozonização. Assim, dentre todos os catalisadores estudados, a hematita dopada com $10 \%$ de manganês em massa mostrou-se mais efetiva para a ozonização catalítica do corante.

\section{REFERÊNCIAS}

ANDERSEN, S. L. F.; FLORES, R. G.; MADEIRA, V. S.; JOSÉ, H. J.; MOREIRA, R. F. P. M. Synthesis and characterization of acicular iron oxide particles obtained from acid mine drainage and their catalytic properties in toluene oxidation. Ind. Eng. Chem. Res., v. 51, p. 767-774, 2012.

DERAZ, N. M.; ALARIFI, A. Novel processing and magnetic properties of hematite/maghemite nano-particles. Ceram. Int., v. 38, p. 4049-4055, 2012.

FLORES, R. G.; ANDERSEN, S. L. F.; MAIA, L. K. K.; JOSÉ, H. J.; MOREIRA, R. F. P. M. Recovery of iron oxides from acid mine drainage and their application as adsorbent or catalyst. J. Environ. Manage, v. 111, p. 53-60, 2012.

JUNG, H.; KIM, J. -W.; CHOI, H.; LEE, J. -H.; HUR, H. -G.; Synthesis of nanosized biogenic magnetite and comparison of its catalytic activity in ozonation. Appl. Catal. B, v. 83, p. 208-213, 2008.

KIRBY, C. S.; DECKER, S. M.; MACANDER, N. K. Comparison of color, chemical and mineralogical compositions of mine drainage sediments to pigment. Environ. Geol., v. 37, p. 243-254, 1999.

LV, A.; HU, C.; NIE, Y.; QU, J. Catalytic ozonation of toxic pollutants over magnetic cobalt and manganese co-doped $\gamma-\mathrm{Fe}_{2} \mathrm{O}_{3}$. Appl. Catal. B, v. 100, p. 62-67, 2010.

MARCELLO, R. R.; GALATO, S.; PETERSON, M.; RIELLA, H. G.; BERNARDIN, A. M. Inorganic pigments made from the recycling of coal mine drainage treatment sludge. $J$. Environ. Manage, v. 88, p. 1280-1284, 2008.

MOUSSAVI, G.; KHOSRAVI, R.; OMRAN, R. 2012. Development of an efficient catalyst from magnetite ore: Characterization and catalytic potential in the ozonation of water toxic contaminants. Appl. Catal. A, v. 445-446, p. 42-49, 2012.

PRADHAN, G. K.; PARIDA, K. M. Dramatic enhancement of catalytic activity over transition metal substituted hematite. J. Ind. Eng. Chem., v. 18, p. 1612-1619, 2012.

REZAEI, E.; SOLTAN, J.; CHEN, N.; LIN, J. Effect of noble metals on activity of $\mathrm{MnO}_{\mathrm{x}} / \gamma-$ alumina catalyst in catalytic ozonation of toluene. Chem. Eng. J., v. 214, p. 219-228, 2013.

RUIHUA, L.; LIN, Z.; TAO, T.; BO, L. Phosphorus removal performance of acid mine drainage from wastewater. J. Hazard. Mater., v. 190, p. 669-676, 2011.

SHEORAN, A. S.; SHEORAN, V. Heavy metal removal mechanism of acid mine drainage in wetlands: A critical review. Miner. Eng., v. 19, p. 105-116, 2006.

VON GUNTEN, U. Ozonation of drinking water: part I. oxidation kinetics and product formation. Water Res., v. 37, p. 1443-1467, 2003.

ZHANG, T.; MA, J. Catalytic ozonation of trace nitrobenzene in water with synthetic goethite. J. Mol. Catal. A, v. 279, p. 82-89, 2008. 